\title{
Adel und Kapitalismus
}

\author{
Die Familie Hohenlohe und der Fürstenkonzern
}

Von Volker Stalmann

Die Adelsforschung erlebt seit einiger Zeit einen beachtlichen Aufschwung. Die Vielzahl der Neuerscheinungen, der Monographien und Tagungsbände zu diesem Thema macht dies deutlich. Wichtige Impulse gingen vor allem von der kulturhistorischen Erweiterung der Sozialgeschichte aus, die das Selbstverständnis und die Mentalität sozialer Gruppen in den Vordergrund rückt und damit Rückschlüsse auf strukturelle Gegebenheiten der Gesamtgesellschaft und Prozesse sozialen Wandels zu ziehen versucht ${ }^{1}$. Zu den kulturgeschichtlich inspirierten neuen Forschungsansätzen zählt dabei die Frage nach dem Verhältnis des Adels zum kapitalistischen Wirtschaftssystem. Konnte der Adel, so die Frage, aufgrund seiner jahrhundertealten Bindung an Grund und Boden seine mental begründeten Reserven gegenüber der industriellen Moderne und der kapitalistischen Wirtschaftsweise überwinden, oder blieb er den tradierten, dem Grundbesitz verbundenen Wirtschaftsformen verhaftet? Gab es also ein adliges Modernisierungsdefizit in dieser Frage?

Wirkungsmächtig waren lange Zeit die Thesen Rosenbergs und Wehlers, für die Adel und Kapitalismus zwei antinome Begriffspaare darstellten. „Im allgemeinen“, so Wehler, „bestand die traditionelle Mentalitätsbarriere gegenüber der industriellen Eigenaktivität unter der Mehrheit der Landadligen“ auch nach 1871 „weiter fort $^{\text {“2 }}$. Der Adel sei letztlich den wirtschaftlichen Herausforderungen der Industriegesellschaft in keiner Weise gewachsen gewesen. Darüber hinaus sei er unfähig gewesen, seinen landwirtschaftlichen Besitz, seinen Großgrundbesitz zumal, zu

1 Vgl. Eckart Conze, Der Adel ist tot - es lebe der Adel! Adelsgeschichte in Deutschland im 19. und 20. Jahrhundert. Entwicklungen und Perspektiven, in: Adel als Unternehmer im bürgerlichen Zeitalter. Vorträge des wissenschaftlichen Kolloquiums der Vereinigten Westfälischen Adelsarchive e.V. vom 28. bis 30. Juli 2004 in Bad Driburg, hg. von Manfred RAscH in Verbindung mit Toni Pierenkemper und Norbert Reimann, Münster 2006, S.49-63; Heinz ReIf, Einleitung, in: Ders. (Hg.), Adel und Bürgertum in Deutschland, 2 Bde., Berlin 2000/2001, hier Bd. 1, S. 7-27.

${ }^{2}$ Hans-Ulrich Wehler, Deutsche Gesellschaftsgeschichte, Bd.3, München 1995, S. 169. 
modernisieren und rentabel zu bewirtschaften. Mit den aufsteigenden bürgerlichen Gutsbesitzern hätten sich die hoch verschuldeten Junker letztlich nicht messen können. Diese am ostelbischen Adel gewonnenen Befunde wurden generalisiert und auf den stark differenzierten und heterogenen deutschen Adel übertragen ${ }^{3}$.

Die neuere Forschung hat dagegen versucht, das ausgesprochen ambivalente Verhalten des Adels gegenüber der modernen marktorientierten Wirtschaft herauszuarbeiten und sowohl die lebensweltlich begründeten mentalen Barrieren und Affekte als auch die Fähigkeit zur flexiblen Anpassung gegenüber der kapitalistischen Wirtschaftsweise aufzuzeigen. Über die um den Landbesitz herum gruppierten gewerblichen Unternehmungen hinaus schien der Adel nur selten die neuen Erwerbschancen des Industriekapitalismus im 19. Jahrhundert wahrgenommen zu haben. Im mentalen Haushalt des Adels schienen Bodenbindung und Risikoaversion, verbunden mit einer gewissen Distanz zur fachlichen Ausbildung, konstitutive Elemente dargestellt zu haben ${ }^{4}$.

Als Beispiel für einen gelungenen Brückenschlag zwischen Adelswelt und Industriekapitalismus gelten in der Regel die Magnaten Oberschlesiens, denen nicht nur Zehntausende von Hektar Land gehörten, sondern die mit ihren Montanunternehmungen auch zu den reichsten und mächtigsten Wirtschaftsführern im Kaiserreich zählten ${ }^{5}$. Sie scheinen am prägnantesten das von den preußischen Junkern dominierte Bild des deutschen Adels zu konterkarieren, eines Adels, der den An-

3 Vgl. ebd., S. 167-176; Hans Rosenberg, Die Pseudodemokratisierung der Rittergutsbesitzerklasse, in: DERs., Machteliten und Wirtschaftskonjunkturen. Studien zur neueren Sozial- und Wirtschaftsgeschichte, Göttingen 1978, S. 83-101, hier S. 90; DERs., Zur sozialen Funktion der Agrarpolitik im Zweiten Reich, in: ebd., S. 102-117.

${ }^{4}$ Vgl. Adel als Unternehmer im bürgerlichen Zeitalter (wie Anm. 1); Hartmut Berghoff, Adel und Industriekapitalismus im Deutschen Kaiserreich. Abstoßungskräfte und Annäherungstendenzen zweier Lebenswelten, in: Heinz ReIf (Hg.), Adel und Bürgertum in Deutschland, Bd. 1: Entwicklungslinien und Wendepunkte im 19. Jahrhundert, Berlin 2000, S. 233-271; Thierry JАсов, Das Engagement des Adels der preußischen Provinz Sachsen in der kapitalistischen Wirtschaft 1860-1914/18, in: ebd., S.273-330; Ilona Buchsteiner, Großgrundbesitz in Pommern 1871-1914. Ökonomische, soziale und politische Transformation der Großgrundbesitzer, Berlin 1993; DIEs., Wirtschaftlicher und sozialer Wandel in ostdeutschen Gutswirtschaften vor 1914, in: Archiv für Sozialgeschichte 36 (1996) S. 85-109; René Schiller, Vom Rittergut zum Großgrundbesitz. Ökonomische und soziale Transformationsprozesse der ländlichen Eliten in Brandenburg im 19. Jahrhundert, Berlin 2003.

${ }^{5}$ Vgl. Toni Pierenkemper, Oberschlesische Magnaten als Unternehmer, in: Adel als Unternehmer im bürgerlichen Zeitalter (wie Anm. 1) S. 131-155; DERS., Unternehmeraristokraten in Schlesien, in: Elisabeth Fehrenbach (Hg.), Adel und Bürgertum in Deutschland 1770-1848, München 1994, S. 129-157; Waclaw Dlugoborski, Die schlesischen Magnaten in der frühen Phase der Industrialisierung Oberschlesiens, in: Toni Pierenkemper (Hg.), Industriegeschichte Oberschlesiens im 19. Jahrhundert. Rahmenbedingungen - Gestaltende Kräfte - Infrastrukturelle Voraussetzungen - Regionale Diffusion, Wiesbaden 1992, S. 107128; Klemens Sківіскі, Industrie im oberschlesischen Fürstentum Pless im 18. und 19. Jahrhundert. Zur ökonomischen Logik des Übergangs vom feudalen Magnatenwirtschaftsbetrieb zum modernen Industrieunternehmen, Stuttgart 2002. 
schluss an die wirtschaftliche Moderne verloren zu haben schien. Die Modernität der schlesischen Magnaten soll im Folgenden am Beispiel der schlesischen Linie des Hauses Hohenlohe und des sogenannten Fürstenkonzerns hinterfragt werden. Konnte der Hochadel sich der kapitalistischen Wirtschaftsweise erfolgreich anpassen, oder spielten nicht doch mentale Reserven in seinem Handeln eine Rolle, die einem erfolgreichen Wirtschaften entgegen standen?

\section{Hohenlohe in Schlesien}

Unter den Magnaten Oberschlesiens nahmen die Fürsten Hohenlohe eine Sonderrolle ein, da sie keine gebürtigen Schlesier waren, sondern Ende des 18. Jahrhunderts durch Einheirat nach Oberschlesien kamen. Die Wurzeln der Familie lagen im deutschen Südwesten. Die Fürstentümer und späteren Standesherrschaften Hohenlohe waren klein, zersplittert und wirtschaftlich rückständig, Gebiete, in denen es kaum Bodenschätze gab und Industrie und Handel unterentwickelt waren $^{6}$. Insofern eröffnete die Heirat des Erbprinzen Friedrich Ludwig zu Hohenlohe-Ingelfingen (1746-1818) mit Gräfin Amalie Marianne von Hoym (1763-1840) im April 1782 dem Haus neue Perspektiven ${ }^{7}$. Denn neben einigen sächsischen Rittergütern brachte Gräfin Hoym die schlesischen Herrschaften Slawentzitz, Birawa, Althammer und Lassowitz in die Ehe ein. Die Linie Hohenlohe-Ingelfingen, die 1805 auch das Erbe des kinderlos verstorbenen Fürsten Hohenlohe-Öhringen antrat, erhielt eine wirtschaftliche Basis.

Die Besitzungen waren industriell erschlossen. Bereits 1620 war in der Herrschaft Slawentzitz ein Werk zur Eisenverhüttung und -verarbeitung errichtet, 1703 in Althammer der erste Hochofen in Betrieb genommen, wenige Jahre später ein

${ }^{6}$ Vgl. Manfred Rasch, Adelige als Unternehmer zwischen Industrialisierung und Ende des deutschen Kaiserreichs. Beispiele aus Württemberg und Baden, in: Eckart Conze/Sönke Lorenz (Hg.), Die Herausforderung der Moderne. Adel in Südwestdeutschland im 19. und 20. Jahrhundert. Viertes Symposion „Adel, Ritter, Ritterschaft vom Hochmittelalter bis zum modernen Verfassungsstaat" (17./18. Mai 2007, Schloss Weitenburg), Ostfildern 2010, S. 83-110, hier S. 100-103, 105-108; Gerhard TAddey, Hohenlohe und Schlesien, in: Hohenlohe in Oberschlesien. Fürsten - Bauern - Bergleute. Historische und volkskundliche Momentaufnahmen 1782-1945. Begleitheft mit Aufsätzen zur Ausstellung des Hauses der Heimat des Landes Baden-Württemberg, Stuttgart 1993, S. 7-15.

7 Friedrich Ludwig, der nach dem Tod seines Vaters 1794 Fürst zu Hohenlohe-Ingelfingen wurde und 1805 das Fürstentum Hohenlohe-Öhringen erbte, sollte als preußischer General noch von sich reden machen. Er war 1806 der Gegner Napoleons bei Jena. Zu Fürst Friedrich Ludwig zu Hohenlohe-Ingelfingen vgl. Günter Richter, Hohenlohe-Ingelfingen, Friedrich Ludwig Fürst zu, in: NDB, Bd. 9, Berlin 1972, S. 489 f.; Richard von Meerheimb, Hohenlohe-Ingelfingen, Friedrich Ludwig Fürst von, in: ADB, Bd. 12, Leipzig 1880, S. 685 f.; Adolf Fischer, Geschichte des Hauses Hohenlohe. Zunächst als Leitfaden beim Unterricht in hohem Auftrag entworfen und den Prinzen und Prinzessinnen des durchlauchtigen Gesammthauses gewidmet, 2. Teil, 2. Hälfte, Stuttgart 1871, S. 282-362.

Zeitschrift für Württembergische Landesgeschichte 74 (2015), S. 201-215.

(C) Kommission für geschichtliche Landeskunde in Baden-Württemberg und Württembergischer Geschichts- und Altertumsverein e.V.

ISSN 0044-3786 
Messinghammer, ein Blechhammer und eine Lüstermanufaktur errichtet worden. Der Aufschwung der Montanindustrie im 18. Jahrhundert wurde wesentlich durch die reichen Erz- und Kohlevorkommen, die umfangreichen Waldbestände wie auch durch den fast kostenlosen Einsatz der frondienstabhängigen Bauernschaft begünstigt $^{8}$.

Lag der Schwerpunkt lange Zeit im Bereich der Eisenindustrie, so wurde die industrielle Basis der oberschlesischen Besitzungen unter Fürst Friedrich Ludwig durch den Kauf des Rittergutes Bittkow 1801 und der Herrschaften Koschentin, Tworog, Landsberg und Czieschowa 1804 und den dazugehörigen Steinkohlegruben erweitert. Mit der Inbetriebnahme eines mit Steinkohlenkoks betriebenen Hochofens ging der Fürst bei der Roheisenproduktion auch neue Wege?.

Einen wichtigen Einschnitt bildete in diesem Zusammenhang die Bauernbefreiung. Die Überwindung der feudalen Agrarverfassung, d.h. die Trennung der jahrhundertealten Verbindung von „Land und Herrschaft“ und damit der Übergang zum Agrarkapitalismus, stellte in den standesherrlichen Gebieten einen sehr langwierigen Prozess dar, der erst unter dem Druck der Revolution von 1848/1849 zu einem Abschluss gelangen konnte. Die mit den Ablösungsgesetzen verbundenen Entschädigungszahlungen setzten die Fürsten in die Lage, sich zu entschulden und das ihnen verbliebene Kapital zum Erwerb von Grundbesitz zu nutzen oder in Industrieunternehmen, in Immobilien oder in Staatsanleihen zu investieren. Die den hohenlohischen Stammesteilen zugesprochenen Entschädigungsbeträge summierten sich schließlich auf 4,4 Millionen Gulden - dies war nach der der Familie Thurn und Taxis gezahlten Summe (5,4 Millionen) die deutschlandweit zweithöchste Entschädigung ${ }^{10}$.

Während Bartenstein und Waldenburg die Entschädigungsgelder in erster Linie zur Tilgung ihrer Schulden verwandten, erwarb Fürst August von Öhringen (1784-1853), der Sohn des Fürsten Friedrich Ludwig, ein ausgedehntes Waldgebiet im Hohenlohischen und vermochte seinen Besitz in Oberschlesien durch den Kauf der Herrschaften Ujest und Bitschin 1838 abzurunden ${ }^{11}$. Der Erwerb von Grund-

${ }^{8}$ Vgl. auch Hohenlohe in Oberschlesien (wie Anm.6), dort der Aufsatz von Gerhard TADDEY, Hohenlohe und Schlesien.

9 Vgl. Taddey (wie Anm. 6) S. 204; Konrad Fuchs, Zur Bedeutung des schlesischen Magnatentums für die wirtschaftliche Entwicklung Oberschlesiens, in: DERs., Beiträge zur Wirtschafts- und Sozialgeschichte Schlesiens, Dortmund 1985, S. 123-152, hier S. 135, insgesamt zur Entwicklung der hohenloheschen Besitzungen in Oberschlesien S. 135-137; Alfons Perlick, Oberschlesische Berg- und Hüttenleute: Lebensbilder aus dem oberschlesischen Industrierevier, Kitzingen/Main 1953, S. 46; ferner Sківіскі (wie Anm. 5) S. 224 f. und insgesamt S. 24-230.

10 Vgl. Walter Demel, Der europäische Adel. Vom Mittelalter bis zur Gegenwart, München 2005, S.110; Hartmut Weber, Die Fürsten Hohenlohe im Vormärz, Schwäbisch Hall 1977, S. 282-290.

${ }^{11}$ Zusammenstellung des Grunderwerbs des Stammesteils Öhringen, in: HZAN GA Seniorat II, Bü 29, Anlage zum Protokoll vom 16./17.9.1839; Weber, Die Fürsten Hohenlohe im Vormärz (wie Anm. 10) S. 282-290. 
besitz besaß für die Fürsten von Hohenlohe damals eindeutig Vorrang vor industriellen Investitionen. Selbst der Sohn des Fürsten August, Prinz Hugo von Hohenlohe-Öhringen, obschon selbst später ein bedeutender Industrieller und Magnat, huldigte ganz den Grundsätzen seines Standes, der sein Selbstverständnis und seine Vorrechte aus seiner Existenz als Grundherr bezog: „Es unterliegt zwar keinem Zweifel, daß sich das in gewerblichen Unternehmungen verwendete Kapital höher verzinst als der Besitz von Grund und Boden, aber ebenso anerkannt ist auch, daß jene Kapitalien umso unsicherer stehen. Ich glaube daher, daß wir an dem Grundsaze, die flüssig gewordenen Fideikommißgelder stets wieder in Grundeigentum anzulegen, als an einer Lebensbedingung des Adels im Wesentlichen jederzeit festhalten müssen und fürchte, dass wir, wenn wir uns erhebliche Abweichungen davon erlauben, auf eine abschüssige Bahn gerathen und der Zersplitterung des Hausvermögens Thür und Thor geöffnet wird." ${ }^{212}$

Diese früh artikulierten Affekte gegenüber der Industrialisierung sollten Hugo jedoch nicht davon abhalten, zum führenden Unternehmer Oberschlesiens und zu einem der bedeutendsten des Kaiserreichs aufzusteigen ${ }^{13}$. Als Zweitgeborener wäre er eigentlich leer ausgegangen, doch sein ältester Bruder Friedrich musste wegen einer nicht standesgemäßen Heirat das Erstgeburtsrecht an seinen Bruder abtreten. Hatte Fürst Hugo anfangs noch von der großen Eisennachfrage im Zuge des Eisenbahnbaus profitieren können, so veranlassten ihn Ende der sechziger Jahre der starke Preisverfall für Eisenwaren infolge der Fertigstellung der Haupteisenbahnlinien und die billige englische und schwedische Konkurrenz, sich sukzessive aus der Eisenherstellung zurückzuziehen und sich auf den Ausbau der Zinkindustrie zu konzentrieren. So ließ er 1871 die Zinkhütte in Bittkow errichten und gliederte ihr 1888 ein Zinkwalzwerk an. Vier Jahre später wurde die Theresien-Zinkhütte in Michalkowitz erworben und drei Jahre darauf die Godulla-Hütte der Gräfin Schaffgotsch gepachtet. In den 1890er Jahren war Fürst Hugo einer der größten Zinkproduzenten der Welt. Mit seinen 41.587 Hektar zählte er ohnehin zu den größten Grundbesitzern Schlesiens. Mit dem Erwerb von Steinkohlegruben seit 1869, der Hoym-Laura-Grube, von Rheinbaben, Fanny, Chassée und der Georgsgrube, sowie dem Ausbau seiner Eisenhüttenwerke seit 1871 verfügte er über ein imponierendes Wirtschaftsimperium ${ }^{14}$.

12 HZAN, Archiv Waldenburg, Domänenkanzlei, Bü 40, Votum des Fürsten Hugo zu Hohenlohe-Öhringen vom 17.4.1854.

${ }^{13} \mathrm{Zu}$ Fürst Hugo zu Hohenlohe-Öhringen und Herzog von Ujest vgl. Alfons Perlick, Hugo Fürst zu Hohenlohe-Öhringen, in: NDB, Bd. 9, S. 492; DERs., Oberschlesische Bergund Hüttenleute (wie Anm. 9) S. 47 f.; Konrad Fuchs, Vom Dirgismus zum Liberalismus. Die Entwicklung Oberschlesiens als preußisches Berg- und Hüttenrevier, Wiesbaden 1970, S. 256; Heinz Gollw itzer, Die Standesherren. Die politische und gesellschaftliche Stellung der Mediatisierten 1815-1918. Ein Beitrag zur deutschen Sozialgeschichte, Stuttgart 1957, S. 258.

14 Vgl. auch Sківіскі (wie Anm. 5) S. 227 f.; Fuchs, Zur Bedeutung des schlesischen Magnatentums (wie Anm. 9) S. 136. 
Wie stark Fürst Hugo bei aller Modernität noch vormodernen, feudalen Werten und Prinzipien verhaftet war, zeigte sein Wunsch nach Standeserhöhung, die 1861 auch Allerhöchsten Orts Berücksichtigung fand. So wurde den oberschlesischen Fideikommissherrschaften des fürstlichen Hauses Hohenlohe-Öhringen der Titel eines Herzogtums verliehen, das fortan den Namen der größten Besitzung Ujest trug. Mit diesem Titel war auch ein Sitz im 1853 gegründeten preußischen Herrenhaus verknüpft ${ }^{15}$.

Als Fürst Hugo 1897 starb, trat sein Sohn Christian Kraft das Erbe an. War Christian Kraft auf die vor ihm liegenden Aufgaben angemessen vorbereitet? Blickt man auf seinen Ausbildungsweg kommen gewisse Zweifel auf. So war dem Besuch der Ritterakademie in Liegnitz zwar 1868 ein zweijähriges Jurastudium an der Universität Bonn gefolgt, das der Aneignung eines juristischen Allgemeinwissens diente. Aber eingehende wirtschaftswissenschaftliche Kenntnisse scheinen ihm nicht vermittelt worden zu sein. Dennoch wird man ihm aufgrund seiner Erfahrungen im Familienunternehmen durchaus unternehmerische Fähigkeiten unterstellen können ${ }^{16}$. Ob er sich jedoch aufgrund seiner anderweitigen Interessen, dem Rennsport und der Politik, kontinuierlich dem Unternehmen widmen konnte, sei dahin gestellt. So gehörte er, wird einmal von seiner erblichen Mitgliedschaft in der württembergischen Ersten Kammer und dem preußischen Herrenhaus abgesehen, mit kurzer Unterbrechung von 1880 bis 1911 dem Reichstag an, wo er die Reihen der Deutschkonservativen verstärkte ${ }^{17}$. Die eigentliche Unternehmensplanung war

${ }^{15} \mathrm{Vgl}$. Hohenlohe in Oberschlesien (wie Anm. 6) S. 11. Vgl. auch GSTA PK Berlin, I. HA, Rep. 89 H, Nr. 30943: Acta betr. die Fideikommiß- und sonstigen Angelegenheiten der Fürsten von Hohenlohe-Oehringen, Herzöge von Ujest und der Prinz von Hohenlohe-Ingelfingen und Hohenlohe-Langenburg, 1825-1915. Ferner das Schreiben von Fürst Hugo zu Hohenlohe-Öhringen vom 31.10.1861 über die Standeserhöhung seiner Herrschaften, in: Staatsarchiv Kattowitz/Polen, Bestand 12/384, Nr. 8985.

16 Zu Fürst Christian Kraft zu Hohenlohe-Öhringen: Alfons Perlick, Christian Kraft Fürst zu Hohenlohe-Öhringen, in: NDB, Bd. 9, Berlin 1972, S. 489; insgesamt: DERs., Oberschlesische Berg- und Hüttenleute (wie Anm. 9) S. 47f.; Fuchs, Zur Bedeutung des schlesischen Magnatentums (wie Anm. 9) S.136f.; Carl Fürstenberg, Die Lebensgeschichte eines deutschen Bankiers 1870-1914, hg. von seinem Sohne Hans Fürstenberg, Berlin 1931, S. 446, zur Gründung der Hohenlohe-Werke AG. Ferner Bernhard Muschol, Die Herrschaft Slawentzitz-Ehrenforst in Oberschlesien: piastisches Kammergut im Spätmittelalter, sächsischer Adelsbesitz und hohenlohesche Residenz in der Neuzeit, Sigmaringen 1993, S. 96-106.

17 Vgl. Perlick, Christian Kraft Fürst zu Hohenlohe-Öhringen (wie Anm. 16); Biographisches Handbuch der württembergischen Landtagsabgeordneten 1815-1933, bearb. von Frank Raberg, Stuttgart 2001, S. 385. Ferner Max Weber, Politik als Beruf. Nachwort von Ralf Dahrendorf, Stuttgart 1992, S. 16 f. Ferner Volker Stalmann, Die Partei Bismarcks. Die Deutsche Reichs- und Freikonservative Partei 1866-1890, Düsseldorf 2000, S. 237-244, 262310, zur Deutschen Reichspartei sowie insgesamt zum Phänomen des Honoratiorenpolitikers; sowie Andreas Biefang, Die andere Seite der Macht. Reichstag und Öffentlichkeit im „System Bismarck“1871-1890, Düsseldorf 2009, S. 162-175.

Zeitschrift für Württembergische Landesgeschichte 74 (2015), S. 201-215.

(C) Kommission für geschichtliche Landeskunde in Baden-Württemberg und Württembergischer Geschichts- und Altertumsverein e.V.

ISSN 0044-3786 
letztlich Aufgabe der hohenlohischen Beamten, die zusammen mit dem Fürsten die großen Linien festlegten und diese auch mit Leben auszufüllen verstanden ${ }^{18}$.

Die hohenlohischen Montanunternehmungen erlebten nach 1897 einen bedeutenden Aufschwung. So wurden die industriellen Besitzungen weiter ausgebaut und die Oheimgrube südlich von Kattowitz sowie die Oehringengrube bei Gleiwitz erworben. Von großer Bedeutung war schließlich 1905 die Umwandlung des Montanbesitzes in eine Aktiengesellschaft, die Hohenlohe-Werke AG. Im Gegenzug erhielt der Fürst eine einmalige Abfindung von 44 Millionen Mark und eine jährliche Rente von 3 Millionen Mark, die 1910 jedoch in einen entsprechenden Aktienanteil umgewandelt wurde. Dadurch wurde Christian Kraft wieder Hauptaktionär und leitete bis zu seinem Tode im Jahre 1926 als Vorsitzender den Aufsichtsrat ${ }^{19}$.

Die Überführung von Industrievermögen in Aktiengesellschaften war ein allgemeiner Prozess, der die Konzentration der Eigentumsverhältnisse in der Region reflektierte. So produzierten seit den 1850er Jahren die vier größten Hütten allein die Hälfte der Zinkproduktion und 1875 die vier größten Werke 62 Prozent der Koksroheisenherstellung. Angesichts des hohen Kapitalbedarfs wurden Aktiengesellschaften bereits in der Mitte des 19. Jahrhunderts ins Leben gerufen: 1853 brachte Graf Henckel von Donnersmarck seine Gruben und Hütten in die Schlesische AG für Bergbau und Zinkhüttenbetrieb ein, 1855 gründete Graf Renard die Minerva AG und 1871 folgte die Vereinigte Königs- und Laurahütte aus dem Vermögen des Grafen von Donnersmarck und die Oberschlesische Eisenbahn-Bedarfs AG. Die Gründung der Hohenlohe-Werke AG stellte in diesem Zusammenhang einen relativ späten Schritt dar ${ }^{20}$.

Zum Aufsichtsratsvorsitzenden wurde 1905 der Bankier Carl Fürstenberg von der Berliner Handels-Gesellschaft gewählt, dem wenig später Carl Klönne von der Deutschen Bank folgte ${ }^{21}$. Vor dem Ersten Weltkrieg zählte die Hohenlohe-Werke AG, die über ein Kapital von 80 Millionen Mark verfügte und knapp 10.000 Arbeiter und Angestellte beschäftigte, zu den bedeutendsten Unternehmen Schlesiens. Die Zinkproduktion erreichte 1913 mit fast 37 Millionen Tonnen mehr als zwanzig Prozent der gesamten oberschlesischen Zinkerzeugung. Dem allgemeinen Preisdruck versuchte man durch den Zusammenschluss der deutschen Zinkproduzenten

18 Vgl. auch Konrad Fuchs, Wirtschaftliche Führungskräfte in Schlesien 1850-1914, in: Zeitschrift für Ostforschung 21 (1972) S. 264-288, hier S. 277.

19 Vgl. HZAN, Archiv Öhringen, Berliner Generalverwaltung, Bü 370: Hohenlohe-Werke AG: Erläuterung der Gesichtspunkte für die Umwandlung des Montan- und Industriebesitzes in Oberschlesien in eine Aktiengesellschaft seitens des Fürsten Christian Kraft zu Hohenlohe-Öhringen, 1905; ferner Fürstenberg (wie Anm.16) S. 445 f.; Erich AchterBERG, Berliner Hochfinanz. Kaiser, Fürsten, Millionäre um 1900, Frankfurt/Main 1965, S. 156-159. Vgl. auch TAdDEY, Die Hohenlohe und Schlesien (wie Anm. 6) S. 210-237.

${ }^{20}$ Pierenkemper, Unternehmeraristokraten in Schlesien (wie Anm. 5) S. 155 f.; DlugoBorski (wie Anm. 5) S. 116.

${ }^{21}$ Vgl. auch Fürstenberg (wie Anm. 16); Achterberg (wie Anm. 19) S. 142-163. 
entgegenzuwirken. Nicht nur in der Zinkerzeugung, sondern auch im Steinkohlebergbau lagen die Hohenlohe-Werke mit einer Jahresförderung von 4 Millionen Tonnen an erster Stelle der privaten oberschlesischen Steinkohlebergwerksunternehmungen ${ }^{22}$.

Diese eindrucksvolle Erfolgsbilanz der Hohenlohe-Werke AG mag als überzeugender Beleg für die Fähigkeit oberschlesischer Hocharistokraten, sich kapitalistische Wirtschafts- und Denkweisen anzueignen, ins Feld geführt werden. Doch ein Blick auf den sogenannten Fürstenkonzern, ein Zusammenschluss verschiedener Wirtschaftsunternehmungen des Fürsten Hohenlohe und des Fürsten Max Egon II. von Fürstenberg, der vor dem Ersten Weltkrieg spektakulär bankrottging, wird zeigen, wie sehr mentale Überhänge und Traditionen auch weiterhin eine nicht unwesentliche Rolle im unternehmerischen Handeln schlesischer Hocharistokraten spielten.

\section{Der Fürstenkonzern}

Die Geschichte des sogenannten Fürstenkonzerns nachzuzeichnen, bereitet einige Schwierigkeiten, da entweder die entsprechenden Unterlagen von den Fürsten Hohenlohe und Fürstenberg noch zu Lebzeiten vernichtet worden sind, oder, sofern sie noch in den Archiven, im Hohenlohe-Zentralarchiv in Neuenstein und im Fürstlich Fürstenbergischen Archiv in Donaueschingen, vorliegen, für die Öffentlichkeit gesperrt sind. Man ist insofern gezwungen, sich der Thematik indirekt zu nähern und mit Hilfe von Lebenserinnerungen der am Trust Beteiligten, von Presseberichten oder anderen aussagekräftigen Quellen, wie Gesandtschaftsberichten, Licht ins Dunkel dieser bedeutenden Adelsunternehmung zu bringen ${ }^{23}$.

Begonnen hatte alles mit einigen Gelegenheitsgeschäften des jüngeren Bruders des Fürsten, des Prinzen Friedrich Karl zu Hohenlohe-Öhringen, der zusammen mit anderen Geldgebern im März 1905 die Madeira AG mit einem Kapital von 3 Millionen Mark gründete. Die AG sollte der Gründung und Unterhaltung eines Sanatoriums und einer Spielbank auf der portugiesischen Insel dienen. Da die anvisierten Projekte sich auf Madeira nicht umsetzen ließen, wurde die Aktiengesellschaft, dessen Kapital mittlerweile auf 15 Millionen Mark angewachsen war, im April 1908 in die neugegründete Handelsvereinigung AG überführt.

$22 \mathrm{Zu}$ den Hohenlohe-Werke AG vgl. Fürstenberg (wie Anm. 16) S. 445 f.; PierenkemPER, Unternehmeraristokraten (wie Anm. 5) S. 149; RAsCH, Adelige als Unternehmer (wie Anm. 6) S. 105 f.; Sкивіскі (wie Anm. 5) S. 230.

${ }_{23}$ Zum Fürstenkonzern vgl. Rasch, Adelige als Unternehmer (wie Anm. 6) S. 105-108; Isabel V. Hull, The Entourage of Kaiser Wilhelm II. 1888-1918, Cambridge 1982, S. 151; Fürstenberg (wie Anm.16) S.445, 498-501; Achterberg (wie Anm.19) S.156-159; Alfred Lansburgh, Die Finanzgeschäfte des Fürstentrust, in: Die Bank, Bd. 2, 1912, S. $223-$ 230.

Zeitschrift für Württembergische Landesgeschichte 74 (2015), S. 201-215.

(C) Kommission für geschichtliche Landeskunde in Baden-Württemberg und Württembergischer Geschichts- und Altertumsverein e.V. 
Die Handelsvereinigung, deren Firmensitz sich in der Berliner Dorotheenstraße befand, war eine Holdinggesellschaft, in die der Fürst Christian Kraft seine Beteiligung an der Levante-Linie, einer Schifffahrtslinie, und sein Vetter Fürst Max Egon II. zu Fürstenberg wiederum seinen aus den Anteilen an der Allgemeinen Berliner Omnibusgesellschaft und der Berliner Terrain- und Baugesellschaft bestehenden Aktienbesitz einbrachten. Die Beteiligungen an der Holding dürften sich zwischen den Fürsten Hohenlohe und Fürstenberg im Verhältnis drei zu zwei bewegt haben. Wegen ihrer hocharistokratischen Gründer wurde die Handelsvereinigung in der Presse auch als „Fürstenkonzern“ oder „Fürstentrust“ bezeichnet.

$\mathrm{Zu}$ den oben genannten Unternehmungen traten bald weitere Engagements wie die Beteiligungen an den Niederlausitzer Kohlenwerken oder den an der Unstrut gelegenen Kaliwerken Friedrichshall und Sarstedt, den Gewerkschaften Reichskrone, Richard und Burggraf. Zur Holding gehörte auch die Palästinabank oder das Berliner Excelsior Hotel und die Deutsche Hotel AG. Neben Obligationen von Terrain-, Schifffahrts- und industriellen Gesellschaften im Wert von 12,2 Millionen Mark und Aktien von Industriegesellschaften im Wert von knapp 20 Millionen Mark verfügte die Handelsvereinigung noch über Staats- und Kommunalpapiere im Wert von über 23,6 Millionen Mark. Der „Fürstenkonzern“ entsprach mithin einem stark diversifizierten Mischkonzern, der einem „Gemischtwarenladen“ glich und sein Engagement auf zahlreiche Felder verteilte, ja zu verzetteln schien. Diese Konzeption resultierte letztlich aus dem Wunsch der Gründer, möglichst rasch hohe Gewinne zu erzielen und dafür auch risikoreiche und hochspekulative Geschäfte einzugehen.

Die Unternehmungen des Fürsten Fürstenberg standen unter keinem guten Stern. So erwarb die Berliner Terrain- und Baugesellschaft, die ursprünglich eine Verwertungsgesellschaft für den Grundbesitz der Berliner Omnibusgesellschaft war, im Norden Berlins Grundstücke und ließ darauf mit einem unvergleichbar hohen Kostenaufwand das „Passage“-Kaufhaus bauen. In Wolf Wertheim, dem ehemaligen Teilhaber des großen A. Wertheimschen Warenhauses, glaubte man nach langer Suche einen Pächter gefunden zu haben. Die weitschweifenden Projekte Wertheims entpuppten sich für die Fürsten jedoch als Fass ohne Boden, in das sie etwa 11 Millionen Mark fließen ließen. Wertheim war jedoch nicht das einzige Problem der Handelsvereinigung. So musste die Vereinigung dem Fürsten Fürstenberg nahezu wertlose Grundstücke am Teltow-Kanal für 8,04 Millionen Mark abkaufen. Überdies musste man von der Deutschen Bank, dafür, dass sie auch weiterhin für die Liquidität des Trusts sorgte, die Beteiligung an der Berliner Baufirma Boswau \& Knauer GmbH übernehmen - einer Baufirma, die durch ihre weitgreifenden Bauprojekte ${ }^{24}$, aber auch durch ihre dubiosen Geschäftspraktiken

${ }^{24}$ Die Baufirma Boswau und Knauer GmbH zeichnete unter anderem für den Bau des Hotels Esplanade, des späteren Theaters am Nollendorfplatz und das spätere Kaufhaus des Westens (KaDeWe) in Berlin verantwortlich. Vgl. RAsCH, Adelige als Unternehmer (wie Anm. 6) S. 107, Anm. 100. 
Schlagzeilen machen sollte. Die Manipulationen der Firma, die nach dem Tode von Hermann Knauer 1909 ans Tageslicht kamen, kosteten den Fürsten Fürstenberg mindestens 5,5 Millionen Mark.

Dieser Misserfolge nicht genug, auch bei den anderen Geschäften stand es nicht zum Besten. Denn die Beteiligung an den Niederlausitzer Kohlenwerken, die Aufschließung der Kalilager an der Unstrut, die Erweiterung der fürstlichen Schifffahrtsinteressen, die Sanierung der Levantelinien und der Ausbau der Palästinabank kosteten Millionen über Millionen. „Der größte Teil der Verluste“, so Manfred Rasch, „ging auf zahllose Beteiligungen (Aktien, Geschäftsanteile etc.) ohne unternehmerischen Schwerpunkt zurück, die ungenügend geprüft sowie mangelhaft geleitet waren und in keinem Verhältnis zu den Eigenmitteln der Handelsvereinigung AG standen“25. Hinzu kam, dass die Struktur der Handelsvereinigung teilweise undurchschaubar war. Die Unternehmungen, so erinnerte sich der Bankier Carl Fürstenberg, seien „so sehr ineinander verschachtelt“ gewesen, „daß hier ein heilloser Wirrwarr entstanden war. [...] Die ganze Sachlage sei unendlich verfahren“ gewesen, „die Zusammenhänge schwer übersichtlich“26.

Da dem Fürsten Fürstenberg nicht die Verfügung, sondern nur die Nutznießung des stattlichen Familienbesitzes zustand und er nur wenig liquide Mittel besaß, war Fürst Hohenlohe gezwungen, seinen Kreditrahmen aufs äußerste anzuspannen und einen Teil seines Vermögens in Bargeld oder Effekten umzuwandeln. In diesem Zusammenhang wurden 1909 und 1911 Kapitalerhöhungen bei der Hohenlohe-Werke AG um 8 bzw. 32 Millionen Mark vorgenommen. Die jährliche Rente des Fürsten über 3 Millionen Mark wurde 1910 in einen entsprechenden Aktienanteil umgewandelt. Aus Protest gegen die durch die vorhandenen Sachwerte nicht gedeckte Kapitalerhöhung auf 80 Millionen Mark und die selbstherrliche Geschäftsführung des Fürsten legten damals die Bankiers Carl Fürstenberg und Carl Klönne ihre Aufsichtsratsmandate nieder. Diesem Schritt folgten auch andere Aufsichtsratsmitglieder wie der oberschlesische Kohlenmagnat Fritz von FriedländerFuld $^{27}$.

Die Lage war so ernst, dass sich schließlich Kaiser Wilhelm II. einschaltete, der mit Fürst Max Egon II. eng befreundet war und mit ihm in Bonn studiert hatte beide gehörten der adligen Studentenverbindung Borussia an. Auf Drängen des Kaisers unternahm die Deutsche Bank unter dem Vorstandsvorsitzenden Arthur von Gwinner den Versuch, den „Fürstenkonzern“ vor dem Zusammenbruch zu retten $^{28}$.

25 Rasch, Adelige als Unternehmer (wie Anm. 6) S. 107.

26 FÜrstenberg (wie Anm. 16) S. 499.

27 Vgl. ebd., S. 497. Zu Fritz Friedländer vgl. auch Achterberg (wie Anm. 19) S. 48-50.

28 Vgl. Fürstenberg (wie Anm.16) S. 499f.; Gwinner geht in seinen Erinnerungen auf diesen Fall nicht ein. Vgl. Arthur von Gwinner, Lebenserinnerungen, Frankfurt/Main 21992. - Der österreichisch-ungarische Botschafter in Berlin, Ladislaus von Szögyenyi-Marich, schrieb Anfang April 1914 nach Wien: „Daß die Mitglieder des Fürstentrusts sich über- 
1912 einigten sich die Fürsten mit der Deutschen Bank auf einen Sanierungsplan. Zuvor hatte bereits Fürst Fürstenberg, als der Minderwert des Teltower Terrains offenkundig wurde, der Gesellschaft 5,25 Mill. Mark gezahlt, wofür seine B-Aktien in vollberechtigte A-Aktien umgewandelt wurden. Man habe den Eindruck, so schrieb damals „Die Bank“, die Monatshefte für Finanz- und Bankwesen, „daß man vor einer Ruine steht, und daß ein Aufwand von 30 oder 50 oder noch mehr Millionen Mark nutzlos vertan worden ist" ${ }^{\text {"29. }}$. Mehrere Unternehmen wurden in der Folge liquidiert und abgewickelt. Ein Großteil, wohlgemerkt die besten Stücke aus dem Kuchen der Berliner Bau- und Terrain-Gesellschaft, konnte zu Geld gemacht werden. Im Juli 1913 standen die beiden Fürsten gleichwohl vor einem Schuldenberg in Höhe von 60 Millionen Mark, in den allerdings die Forderungen der Deutschen Bank noch nicht eingerechnet sind ${ }^{30}$.

Die Lage nahm sich zwischenzeitlich so katastrophal aus, dass Christian Kraft sogar ohne Wissen der Bankgläubiger ein bedeutendes Effektendepot nach London schicken ließ, um für den Fall des Zusammenbruchs einen Notgroschen im Ausland zu besitzen. Aber in den folgenden Jahren gelang es dem Fürsten mit Hilfe seines fähigen Kammerpräsidenten von Kleefeld, dem Schwager des späteren Reichskanzlers Stresemann, sich aus der Abhängigkeit von der Deutschen Bank zu lösen und finanzielle Unterstützung bei der damals noch unbedeutenden Nationalbank für Deutschland, dessen Verhandlungsführer kein geringerer als Hjalmar Schacht war, zu erhalten. Die Nationalbank schloss sich in den zwanziger Jahren mit der Darmstädter Bank zusammen und wurde wenige Jahre später im Zuge der Bankenkrise von der Dresdner Bank geschluckt. Für den Konzern war auch entscheidend, dass er im tschechischen Kohlenmagnaten Ignaz Petschek (Aussig) einen wichtigen wirtschaftlichen Partner fand und die nach dem Zusammenbruch des Fürstenkonzerns noch verbliebenen wirtschaftlichen Unternehmungen zu einem halbwegs vernünftigen Preis verkaufen konnte, so dass sich die wirtschaftliche Lage der Hohenlohe-Werke vor dem Krieg wieder erträglicher gestaltete. Aus den wirtschaftlichen Schwierigkeiten half dem Fürsten allerdings erst die In-

haupt noch in dieser Weise vor dem vollkommenen Ruin retten konnten, verdanken sie eigentlich vor Allem der Initiative Seiner Majestät Kaiser Wilhelms, der, um Seinen Freund den Fürsten Fürstenberg zu retten, den Director der Deutschen Bank Herrn von Gwinner zu Sich kommen ließ und ihm den Auftrag erteilte, die Angelegenheit der Liquidierung des Fürstentrusts in die Hand zu nehmen und für sich und die fürstlichen Partner so viel zu retten, als überhaupt noch möglich sei.“ Bericht des österreichisch-ungarischen Botschafters von Szögyenyi-Marich nach Wien, 8. April 1914, in: HHStA Wien, PA III 171.

${ }^{29}$ Zum Fürstenkonzern vgl. Rasch, Adlige als Unternehmer (wie Anm. 6) S. 105-108; Alfred Lansburgh, Die Finanzgeschäfte des Fürstentrust, in: Die Bank, Bd. 2, 1912, S. $223-$ 230, hier S. 227. Vgl. auch Deutsche Bank Archiv, Frankfurt, Akte „Hohenlohe-Werke S 461“, Zeitungsmappe SG 10/005 sowie PO 1251 (Anleihe für die Hohenlohe-Werke AG von 1912).

30 Vgl. Rasch, Adelige als Unternehmer (wie Anm. 6) S. 107. 
flation der zwanziger Jahre, durch die der Schuldenberg umstandslos getilgt werden konnte ${ }^{31}$.

Der Zusammenbruch des Handelsvereinigung AG führte zu heftigen Auseinandersetzungen zwischen den beiden Fürsten. „Alle Versuche“, so berichtete der württembergische Gesandte in Berlin, von Varnbüler, im Februar 1914 nach Stuttgart, „dem drohenden Proceß über die zwischen den Fürsten Fürstenberg und Hohenlohe-Oehringen bei Auflösung des Fürstenconcerns sich ergebenden Differenzen durch Vergleich vorzubeugen, sind bisher gescheitert. Fürst Hohenlohe hatte seine vermeintliche Forderung von circa 30 Millionen gegen seinen Vetter auf die Hälfte ermäßigt, dieser aber nur eine 20jährige Ratenzahlung von je $400.000 \mathrm{M}$ geboten, die überdies von Seiten eines Fideicommißbesitzers, der jederzeit zu Gunsten seines Sohnes abdanken könnte, nicht gesichert erschien. - Der von Hohenlohe ausgehende und auch von dem Rechtsbeistand der Gegenpartei, dem für sehr anständig geltenden Justizrat von Gordon befürwortete Vorschlag, die Entscheidung einem Schiedsrichter zu unterwerfen, etwa dem Herrn Reichskanzler unter sachverständiger Assistenz des Präsidenten der Reichsbank Havenstein, wurde von Fürstenberg persönlich abgelehnt. Nun ist, als letztes Mittel zur Vermeidung eines Processes, der bei der - gelinde gesagt - Dunkelheit der zu Grunde liegenden und aufzuklärenden Geschäftsgebarungen der Generalbevollmächtigten beider Parteien, nicht nur für diese höchst compromettant werden, sondern bei der bekannten Freundschaft des Kaisers für Fürstenberg auch politisch ausgebeutet werden könnte, neuerdings beabsichtigt, Seiner Majestät die Sachlage vorzutragen und anheimzugeben, Allerhöchst Seinen Einfluß zu Gunsten eines Vergleichs geltend zu machen. Es erscheint dies umso mehr geboten, als Seine Majestät bisher des Glaubens ist, daß Fürst Fürstenberg nur durch seinen Vetter Hohenlohe in diese wilden Speculationen hereingezogen worden sei, während die große Mehrheit der haute finance die entgegengesetzte Ansicht vertritt und auch durchaus unparteiische und vertrauenswerte Männer unserer Gesellschaftskreise auf Grund ihnen vorgelegter authentischer Schriftstücke die Handlungsweise des Fürsten Fürstenberg mit seinen Standesbegriffen für unvereinbar halten.“32

Ein Vergleich zwischen beiden Fürsten kam Anfang April zustande. Fürst Fürstenberg schied aus der Handelsvereinigung und verfügte seitdem über kein freies Vermögen mehr. Als alleiniger Gesellschafter war Christian Kraft mit Hilfe eines ihm von der Deutschen Bank gewährten Kredits über 80 Millionen Mark seitdem bemüht, die verschiedenen Unternehmungen abzuwickeln. Als Deckung des Kredits diente neben dem Immobiliarvermögen der Handelsvereinigung der unga-

31 Denkschrift Richard Chrambachs über „Die wirtschaftlichen Zusammenhänge und finanzielle Entwicklung des Haus-Vermögens Hohenlohe-Öhringen in den Jahren 1932 und 1937“, um 1937, in: HZAN, Archiv Öhringen, Berliner Generalverwaltung, Bü 4.

${ }^{32}$ Bericht des königlichen württembergischen Gesandten in Berlin, von Varnbüler, an den Staatsminister der auswärtigen Angelegenheiten, Dr. von Weizsäcker, Berlin, 27.2.1914, in: HStA Stuttgart E 50/03, Bü 208. 
rische Besitz des Fürsten. „Die Deutsche Bank“, so berichtete der österreichisch-ungarische Botschafter in Berlin, Ladislaus von Szögyenyi-Marich, im April nach Wien, „die, wie es heißt, einen Teil der notwendigen Sanierungen selbst in die Hand genommen hat - ist freilich genügend sichergestellt, zumal sie sich auch in den Hohenlohe-Werken eine entscheidende Ingerenz in der Generalversammlung so gut wie gesichert hat. - Eine andere Frage ist es, in welcher Weise Fürst Hohenlohe der nötigen Verzinsung seiner nicht weniger als 110 Millionen M. betragenden Passiven zu begegnen wissen wird; denn wenn sein freies Vermögen auch mit einiger Verläßlichkeit auf ca. 130 Millionen M. geschätzt werden kann, so besteht dasselbe doch nur zu einem Teil aus Gütern, die niemand reich genug ist zu kaufen, zum anderen aus vierten Hypotheken, Anteilen an schlecht gehenden Hôtels und Warenhäusern (Wolf Wertheim). Daß sein wertvolles, großes Unternehmen, die Hohenlohe-Werke, sich in einem Stadium der Reorganisation befindet, in welchem es für die nächste Zukunft wenig Ertrag verheißt, ist für den Fürsten wohl das größte Verhängnis. Vielleicht, daß es jetzt, wo der Vergleich mit dem Fürsten Fürstenberg die Möglichkeit der Einflußnahme auf die einzelnen Tochtergesellschaften der Handelsvereinigung eröffnet, gelingen wird, wenigstens die Leitung dieser, in recht erbarmungswürdigem Zustand befindlichen Unternehmungen zu reorganisieren und dadurch den Verkauf der ungarischen Güter überflüssig zu machen, der bei der gegenwärtigen Lage zu günstigen Bedingungen kaum durchführbar sein würde.“33

Zum 25. April 1914 wurde die Handelsvereinigung AG liquidiert ${ }^{34}$. Die Verluste, die Fürst Christian Kraft durch den Zusammenbruch der Handelsvereinigung erlitt, waren mit etwa 110 Millionen Mark erheblich. Die Verbindlichkeiten wurden später auf die Nationalbank für Deutschland überführt und letztlich erst durch die Inflation der zwanziger Jahre abgelöst ${ }^{35}$.

Die Baronin von Spitzemberg berichtet unter dem 14. Dezember 1913 in ihrem Tagebuch, dass ihr Sohn, Lothar Hugo, der acht Tage auf Jagd beim Fürsten gewesen sei, „tief ergriffen von dem letzten Akte einer Tragödie, den er erlebte“, gewesen sei. „Bei dem Zusammenbruche des ,Fürstenkonzerns' hat Christian Kraft so viel verloren, daß er sich aufs äußerste einschränken muß, seinen Hofhalt in Slawentzitz auflöst und hofft, in Ungarn sich ein kleines Anwesen aufrechtzuerhalten. Ganz bewußt hat er seine Freunde zum letzten Male eingeladen und rührend von ihnen als Jagdherr Abschied genommen. Nun wird alles abgeschossen, die Jä-

33 Bericht des österreichisch-ungarischen Botschafters in Berlin, von Szögyenyi-Marich, an Außenminister Leopold Graf Berchtold, 8. April 1914, in: HHSTA Wien, PA III 171.

${ }^{34}$ Vgl. Handbuch der Deutschen Aktien-Gesellschaften, Ausgabe 1916/17, Bd. 2, S. 244.

35 Vgl. den Bericht des österreichisch-ungarischen Botschafters in Berlin, von Szögyenyi-Marich, vom 8. April 1914 (wie Anm. 33); ferner die Aktennotiz vom 28.12.1933, in: Dresdner Bank-Archiv, Berlin, Akte 30201-2001. BE; sowie die Denkschrift „Die wirtschaftlichen Zusammenhänge und finanzielle Entwicklung des Haus-Vermögens Hohenlohe-Oehringen in den Jahren 1932-1937“, besonders S.1-6, in: Hohenlohe-Zentralarchiv Neuenstein, Archiv Öhringen, Generalverwaltung, Bü 4. 
gerei entlassen, Hunderte dabei in Not versetzt - im Grunde unverantwortlich, daß ein Herr wie Hohenlohe soweit kam durch eigene Unfähigkeit, die Wahl ganz unfähiger Beamter, letztens allerdings durch die versuchte Sanierung Fürstenbergs. “ 36

Ob der Zusammenbruch des Fürstenkonzerns Fürst Christian Kraft ruiniert hat, ist fraglich. So schätzte Rudolf Martin, Regierungsrat im Reichsamt des Innern, das Vermögen des Fürsten 1914 in seinem Jahrbuch der Millionäre auf 154 Millionen Mark und sein Einkommen auf 6,5 Millionen Mark. Doch bleiben Zweifel, ob angesichts der bestehenden Schulden diese Schätzung der Wirklichkeit entsprach. Mit diesem Vermögen wäre der Fürst in Preußen hinter Bertha Krupp von Bohlen und Halbach (283 Millionen Mark Vermögen) und noch vor Kaiser Wilhelm II. (140 Millionen Mark) der zweitreichste Mann gewesen. In Oberschlesien war er zudem mit seinen knapp 43.000 Hektar der größte Grundbesitzer. In Württemberg übertraf ihn 1914 allein Fürst Albert von Thurn und Taxis mit einem Vermögen von 270 Millionen und einem Einkommen von 5 Millionen Mark. Auf Rang drei hatte Christian Kraft dagegen Fürst Max Egon zu Fürstenberg verwiesen (110 Millionen Mark Vermögen, 2,1 Millionen Mark Einkommen) ${ }^{37}$.

Was den Fürsten Hohenlohe und Fürstenberg gefehlt habe, so urteilte der Bankier Carl Fürstenberg rückblickend, „war der industrielle Fernblick eines Henckel-Donnersmarck oder, in Ermangelung dessen, eine Anlehnung an die ersten Fachleute der Zeit, die gerade den Fürsten ihren Rat sicherlich gern zur Verfügung gestellt hätten. Hierzu kam es jedoch leider nicht oder doch erst in einem Zeitpunkt, als es schon zu spät war. Fürst Fürstenberg stützte sich während des ganzen ersten Teils seiner geschäftlichen Expansion auf die Ratschläge des Bankiers N. ${ }^{38}$, dem ich eine finanzielle Begabung zwar nicht absprechen möchte, der aber für diese Aufgabe sicherlich nicht der richtige Mann war. “39

In der Presse wurde man deutlicher. So urteilte ein Journalist über den Fürsten Fürstenberg: „Seine Ratgeber besitzen eine fast unumschränkte Vollmacht in geschäftlichen Dingen, und während er weite Reisen unternimmt, werden auf seinen Namen die riskantesten Finanztransaktionen vorgenommen, Geschäfte seltsamster Natur abgeschlossen, Kreditoperationen größten Stils durchgeführt, die er persönlich vielleicht kaum zu verstehen imstande wäre, gäbe er sich selbst die Mühe, sie kennen zu lernen. Dieser angeblich so geschäftstüchtige Magnat, der

${ }^{36}$ Das Tagebuch der Baronin Spitzemberg: Aufzeichnungen aus der Hofgesellschaft des Hohenzollernreiches. Hildegard von Spitzemberg, ausgewählt und herausgegeben von Rudolf Vierhaus, Göttingen ${ }^{5} 1989$, S. 565.

37 Vgl. Rudolf Martin, Jahrbuch des Vermögens und Einkommens der Millionäre in der Provinz Schlesien, Berlin 1913, S.2, 5 f., 100, 138, 197; Willi A. Boelcke, Millionäre in Württemberg. Herkunft - Aufstieg - Traditionen. Mit einem Faksimile-Abdruck des „Jahrbuchs des Vermögens und Einkommens der Millionäre in Württemberg mit Hohenzollern“ von Rudolf Martin, 1914, Stuttgart 1997, S. 10, 24 f., 92, 239, 247 f., 269, 282.

38 Gemeint ist der Bankier Karl Neuburger.

39 Fürstenberg (wie Anm. 16) S. 498. 
,fürstliche Finanzmann', ist im Grunde das reinste Kind in allen geschäftlichen Angelegenheiten. ${ }^{\text {"40 }}$

Wenn man nach den Gründen für den spektakulären Zusammenbruch des Fürstenkonzerns fragt, so wird man in der Tat die geradezu beiläufige Art und Weise, mit der die Fürsten in wirtschaftspolitischer Hinsicht agierten, als Erklärung heranziehen müssen. Hohenlohe stand zwar seit 1897 einem florierenden Unternehmenskomplex vor, verfügte jedoch über keine fundierte wirtschaftswissenschaftliche Ausbildung. Es muss bezweifelt werden, ob ihm die Problematik der Handelsvereinigung, die einen Zusammenschluss unterschiedlichster Unternehmungen ohne inneres, stimmiges Konzept darstellte, bewusst war, wenn er überhaupt den Überblick über die verschiedenen Engagements des in sich verschachtelten Konzerns bewahren konnte. Hinzu trat die unglückliche Auswahl des Personals, der Berater und Geschäftsführer, bei der offensichtlich persönliche Sympathien eine größere Rolle als fachliche Kompetenz gespielt haben dürften. Insgesamt scheint das Beispiel des Fürstenkonzerns auch bei den schlesischen Magnaten mentale Dispositionen und Verhaltensweisen aufzuzeigen, die eine gewisse Distanz zur industriellen Welt und den Anforderungen einer komplexen Arbeitswelt offenbaren. Durch die Professionalisierung der Unternehmensstrukturen schienen auch die Magnaten Oberschlesiens ins Hintertreffen zu geraten.

„Wo die Gründe für dieses einzigartige Débacle, für diese Fürstendämmerung, zu suchen sind“, so urteilte der österreichisch-ungarische Botschafter von Szögyenyi-Marich, „welche zwei der größten Feudalvermögen der deutschen Hocharistokratie so rasch zunichte machen konnte, so sind sie wohl nicht schwer zu bezeichnen: sie werden weniger in den geschäftlichen Conjuncturen, auch nicht in einem allgemeinen wirtschaftlichen oder politischen Niedergang zu suchen sein, als vielmehr darin, daß heutzutage die nutzbringende Verwaltung und geschäftliche Verwertung so colossaler Einzelvermögen - soweit sie nicht fideicommissarisch geschützt sind - eine ernste Hingabe und Vertiefung erfordert, deren blosser Dilettantismus nicht fähig sein kann “41.

40 Princeps: Fürst Fürstenberg, in: Die Standarte, 5. Jahrgang, Nr. 23, 14.3.1911, S. 5, insgesamt S. 1-5.

${ }^{41}$ Bericht des österreichisch-ungarischen Botschafters in Berlin, von Szögyenyi-Marich, an den Außenminister Leopold Graf Berchtold, 8. April 1914, in: HHSTA Wien, PA III 171. 